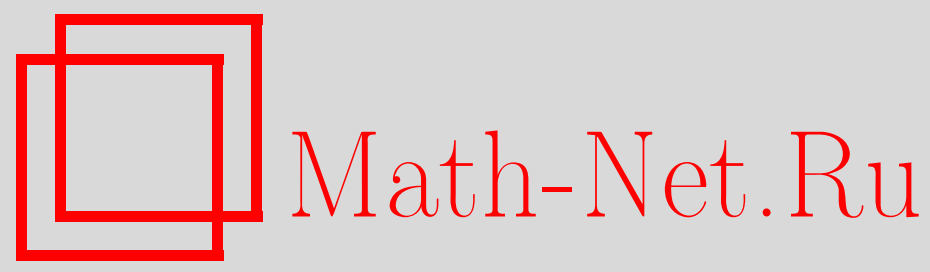

Э. Э. Шноль, Об одной экстремальной задаче про вероятностные распределения, Матем. заметки, 2002, том 71, выпуск 6, 937-945

DOI: https://doi.org/10.4213/mzm398

Использование Общероссийского математического портала Math-Net.Ru подразумевает, что вы прочитали и согласны с пользовательским соглашением http://www . mathnet.ru/rus/agreement

Параметры загрузки:

IP : 3.81 .55 .215

26 апреля 2023 г., 09:31:09

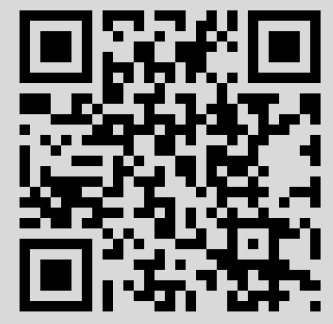




\title{
ОБ ОДНОЙ ЭКСТРЕМАЛЬНОЙ ЗАДАЧЕ ПРО ВЕРОЯТНОСТНЫЕ РАСПРЕДЕЛЕНИЯ
}

\author{
Э.Э. Шноль
}

Заданная плотность распределения вероятностей модифицируется посредством умножения на все положительные функции с фиксированным отношением верхней и нижней грани. Произведения нормируются так, чтобы получить модифицированные плотности вероятностей. Изучаются значения дисперсии в полученном классе вероятностных распределений. Показано, что верхняя грань дисперсии достигается для кусочно-постоянной модифицирующей функции, имеющей вид "прямоугольной ямы". Аналогичное утверждение справедливо для минимума дисперсии. Показано, что в этом классе есть единственное распределение с максимальной дисперсией.

Библиограффия: 0 названий.

1. Постановка задачи. Следующий вопрос возник при рассмотрении простейших математических моделей генетики популяций (и был предложен автору генетиком А. С. Кондрашовым).

Пусть задано распределение вероятностей, имеющее плотность $p_{0}(x)$. Образуем новую плотность $p(x)$, умножив $p_{0}(x)$ на положительную функцию $w(x)$ (с последующей нормировкой). Вопрос: насколько сильно может при этом измениться дисперсия? Главное ограничение (делающее вопрос содержательньп) - “размах" $w(x)$ конечен, т.е.

$$
\sup w(x) \leqslant K \inf w(x) .
$$

Естественно, предполагается, что исходное распределение $p_{0}(x)$ имеет (конечные) среднее значение и дисперсию. В дальнейшем мы будем считать, что среднее значение равно нулю, а дисперсия - единище.

Обозначим через $I_{k}[w]$ следующие функционалы:

$$
I_{k}[w]=\int_{-\infty}^{+\infty} s^{k} w(s) p_{0}(s) d s, \quad k=0,1,2 .
$$

Модифицированная плотность определяется по формуле $p(x)=w(x) p_{0}(x) / I_{0}[w]$.

ЗАДАЧА. При заданной непрерывной плотности вероятностей $p_{0}(x)$ и заданной постоянной $K(K>1)$ указать положительные (измеримые) функиии $w(x)$, на которых достигается верхняя и нижняя грань дисперсии распределения $p(x)$ функиионала $D[w]-$ при ограничении (1.1). Здесь

$$
D[w]=\frac{I_{2}[w]}{I_{0}[w]}-(M[w])^{2}, \quad M[w]=\frac{I_{1}[w]}{I_{0}[w]} .
$$


Ответ представляется интуитивно ясньм. Чтобы получить максимальную дисперсию, нужно в наибольшей степени (насколько позволяет ограничение (1.1)) увеличить вероятности "далеких" значений $x$. А чтобы получить наименьшую дисперсию, наоборот, уменьшить вероятности “далеких" значений $x$. Точная формулировка и доказательство соответствующей теоремы составляет предмет этой заметки.

2. Ступенчатые функции. Функционалы (1.3) не меняются при умножении $w(x)$ на константу. Вместо (1.1) будем использовать следующие ограничения (первое - для поиска максимума $D$, второе - для минимума):

$$
\begin{gathered}
1+\gamma \leqslant w(x) \leqslant 1, \quad-1<\gamma<0, \quad K=(1+\gamma)^{-1}, \\
1 \leqslant w(x) \leqslant 1+\gamma, \quad \gamma>0, \quad K=1+\gamma .
\end{gathered}
$$

Следующие кусочно-постоянные функции будем называть ступенчатыми:

$$
\begin{array}{ll}
w(x)=1 & \text { при } x \leqslant a \text { и } x \geqslant b, \\
w(x)=1+\gamma & \text { при } a<x<b .
\end{array}
$$

На семействе (2.3) функционалы $I_{k}, M$ и $D$ превращаются в функции двух переменных. В частности (см. (1.2)),

$$
\begin{aligned}
& I_{k}(a, b)=1+\gamma \int_{a}^{b} x^{k} p_{0}(x) d x, \quad k=0,2, \\
& I_{1}(a, b)=\gamma \int_{a}^{b} x p_{0}(x) d x .
\end{aligned}
$$

При $a \rightarrow-\infty$ или $b \rightarrow+\infty$ все эти функции стремятся к пределам - непрерьвньг функциям одной переменной. Точнее, естественная область определения функций $I_{k}(a, b)$, $M(a, b)$ и $D(a, b)$ (в которой они непрерывны) - полуплоскость $a \leqslant b$, пополненная прямыми $a=-\infty$ и $b=+\infty$ (Это компактное множество можно представлять себе в виде треугольника, положив, например, $\widetilde{a}=\varphi(a), \widetilde{b}=\varphi(b), \varphi(s)=s /\left(1+s^{2}\right)^{1 / 2}$.)

\section{3. Формулировка основного результата.}

ТЕОРема 1. Пусть функиия $p_{0}(x)>0$ непрерывна при всех $x$. Тогда

1) верхняя грань функиионала $D[w]$ на множестве (измеримых) функиий $w(x)$, удовлетворяющих неравенствам (2.1), достигается на ступенчатой функиии вида (2.3) с конечными а и b; әкстремальная ступенчатая функиия единственна;

2) нижняя грань функиионала $D[w]$ на множестве функиий $w(x)$, удовлетворяющих неравенству (2.2), достигается на ступенчатой функции вида (2.3) с конечными а и $b$.

Сформулированная теорема сводит экстремальную задачу для функционала $D[w] \mathrm{k}$ нахождению экстремумов функции $D(a, b)$.

ЗАмечАниЕ 1. Ступенчатая функция, доставляющая нижнюю грань $D[w]$, не обязательно единственна. 
4. Экстремумы функции $D(a, b)$. Убедимся сначала, что, используя для модификации плотности ступенчатые функции с $\gamma<0$, можно увеличить дисперсию: $\sup D(a$, b) $>1$.

По определению для ступенчатой функции $w(x)$ имеем

$$
\begin{array}{ll}
p(x)=N(1+\gamma) p_{0}(x) & \text { при } a<x<b, \\
p(x)=N p_{0}(x) & \text { при } x<a \text { и } x>b,
\end{array} \quad N=\frac{1}{I_{0}[w]} .
$$

При $a<b<\infty$ (и $\gamma<0)$ вьполнены неравенства $1+\gamma<I_{0}[w]<1$, и потому $N>1$, а $N(1+\gamma)<1$. Таким образом, при отрищательном $\gamma p(x)$ меньше $p_{0}(x)$ в интервале $(a, b)$ и больше $p_{0}(x)$ вне этого интервала. Выберем теперь $a$ и $b$ так, чтобы при переходе от $p_{0}(x)$ к $p(x)$ среднее не менялось. По предположению среднее значение распределения $p_{0}(x)$ равно нулю. Поэтому для всякого $a<0$ существует $b>0$ такое, что

$$
\int_{a}^{b} x p_{0}(x) d x=0
$$

Выбрав такие $a$ и $b$, мы получим $M(a, b)=0$ (при любом $\gamma)$. Поскольку среднее не изменилось, а вероятности более далеких от среднего значений увеличились, то ясно, что дисперсия распределения $p(x)$ больше исходной. Проверим это утверждение выкладкой. В силу сказанного выше о знаке $p(x)-p_{0}(x)$ имеем

$$
\int_{-\infty}^{\infty}(x-a)(x-b)\left[p(x)-p_{0}(x)\right] d x>0 .
$$

Поскольку средние значения распределений $p$ и $p_{0}$ равны нулю, получим $D(a, b)>1$ ( $a<0$ можно выбрать произвольно).

Вполне аналогично при $\gamma>0$ можно получить $D(a, b)<1$. Выпишем производные функции $D$ (см. (1.3) и (2.4); аргументы у функций $D(a, b)$ и $I_{k}(a, b)$ опущены):

$$
\begin{aligned}
& \frac{\partial D}{\partial a}=-\frac{\gamma p_{0}(a)}{I_{0}^{2}}\left\{a^{2} I_{0}-I_{2}-2 \frac{I_{1}}{I_{0}}\left(a I_{0}-I_{1}\right)\right\}, \\
& \frac{\partial D}{\partial b}=\frac{\gamma p_{0}(b)}{I_{0}^{2}}\left\{b^{2} I_{0}-I_{2}-2 \frac{I_{1}}{I_{0}}\left(b I_{0}-I_{1}\right)\right\} .
\end{aligned}
$$

Пусть сначала $\gamma<0$. При фиксированном $b$ и $a \ll-1$ производная по $a$ положительна, поскольку первое слагаемое в скобке мажорирует остальные: максимум $D(a, b)$ на каждой прямой $b=\bar{b}$ (включая $\bar{b}=\infty$ ) достигается при конечном $a$. Аналогично, максимум $D(a, b)$ на каждой прямой $a=\bar{a}$ достигается при конечном $b$. Так как $D(b, b) \equiv 1$, то $\sup D(a, b)$ достигается во внутренней точке. Приравняв к нулю частные производные (4.1), получим (поскольку $p_{0}(a)$ и $p_{0}(b)$ отличны от нуля) уравнения, связывающие $a$ и $b$ в экстремальной точке со значениями $I_{k}$ в этой точке:

$$
\frac{I_{1}\left(a_{*}, b_{*}\right)}{I_{0}\left(a_{*}, b_{*}\right)}=\frac{1}{2}\left(a_{*}+b_{*}\right), \quad \frac{I_{2}\left(a_{*}, b_{*}\right)}{I_{0}\left(a_{*}, b_{*}\right)}=\frac{1}{2}\left(a_{*}^{2}+b_{*}^{2}\right) .
$$

Повторяя рассуждения, получим для $\gamma>0$ : нижняя грань $D(a, b)$ достигается во внутренней точке $\left(a_{*}, b_{*}\right)$ и при этом вьполнены соотношения (4.2). 
ЗАмЕчАниЕ 2. Среднее значение и дисперсия, отвечающие экстремальной ступенчатой функции, такие же, как у дискретной случайной величины, принимающей значения $a_{*}$ и $b_{*}$ с вероятностью $1 / 2$.

5. Доказательство первого утверждения теоремы 1. Пусть $w_{*}(x)$ - ступенчатая функция с $\gamma<0$, для которой вьполнены соотношения (4.2), а $w(x)$ - произвольная функция, для которой вьполнены неравенства (2.1). Рассмотрим интеграл

$$
\int_{-\infty}^{+\infty}\left(w_{*}(x)-w(x)\right)\left(x-a_{*}\right)\left(x-b_{*}\right) p_{0}(x) d x
$$

Этот интеграл неотрицателен, поскольку подьнтегральная функция везде неотрицательна. Он обращается в нуль, только если $w_{*}(x) \equiv w(x)$ (почти всюду). Обозначив $I_{k *}=I_{k}\left[w_{*}\right]$ и $I_{k}=I_{k}[w]$, запишем

$$
I_{2 *}-I_{2}-\left(a_{*}+b_{*}\right)\left(I_{1 *}-I_{1}\right)+a_{*} b_{*}\left(I_{0 *}-I_{0}\right) \geqslant 0 \text {. }
$$

Выразив $a_{*}+b_{*}$ и $a_{*} b_{*}$ из $(4.2)$ и поделив на $I_{0}$, получим

$$
-\frac{I_{2}}{I_{0}}+2 \frac{I_{1 *}}{I_{0 *}} \frac{I_{1}}{I_{0}}-2\left(\frac{I_{1 *}}{I_{0 *}}\right)^{2}+\frac{I_{2 *}}{I_{0 *}} \geqslant 0
$$

или (см. (1.3))

$$
D\left[w_{*}\right] \geqslant D[w]+\left(M\left[w_{*}\right]-M[w]\right)^{2} .
$$

Равенство здесь возможно лишь при $w_{*}(x) \equiv w(x)$ (почти всюду).

СлЕдСТВИЕ. Имеется только одна ступенчатая функиия, на которой достигается верхняя грань функиионала $D[w]$.

Действительно, во всяком локальном максимуме функции $D(a, b)$ вьполняются соотношения (4.2). При двух максимумах получились бы противоречивые неравенства $D\left[w_{*}^{(1)}\right]>D\left[w_{*}^{(2)}\right]$ и $D\left[w_{*}^{(2)}\right]>D\left[w_{*}^{(1)}\right]$.

ЗАмЕчАнИЕ 3 . Если распределение $p_{0}(x)$ симметрично (т.е. $p_{0}(-x)=p_{0}(x)$ ), то экстремальная ступенчатая функция тоже симметрична. Если же $p_{0}(x)$ не симметрично, то, вообще говоря, для получения максимальной дисперсии нужно изменить среднее значение.

6. Вспомогательная задача. Перейдем к более трудному вопросу о минимуме дисперсии. Рассмотрим функции (2.3) при $\gamma>0$. Пусть при $a=a_{*}, b=b_{*}$ достигается минимум $D(a, b)$, и пусть $w_{*}(x)$ - соответствующая ступенчатая функция. Рассмотрим произвольную функцию $w(x)$, удовлетворяющую неравенствам (2.2). Подьнтегральное выражение в (5.1) теперь неположительно, и вместо (5.2) мы получим

$$
D\left[w_{*}\right] \leqslant D[w]+\left(M\left[w_{*}\right]-M[w]\right)^{2} .
$$

В отличие от задачи на максимум, (6.1) доставляет нужное неравенство лишь при $M[w]$ $=M\left[w_{*}\right]$. Однако формула (6.1) подсказывает, что полезно рассмотреть следующую вспомогательную задачу. 
ВСПОМОГАТЕЛЬНАЯ ЗАДАЧА. Найти функиии $w(x)$, удовлетворяющие неравенству (2.2), на которых достигается нижняя грань функционала $D[w]$ при заданном среднем значении $M[w]=m$.

Предварительно рассмотрим функционал $M[w]$ на ступенчатых функциях (2.3).

7. Экстремумы $M(a, b)$. Напишем соотношения (опустив аргументы у $M$ и $I_{k}$ )

$$
\frac{\partial M}{\partial a}=-\frac{\gamma p_{0}(a)}{I_{0}^{2}}\left(a I_{0}-I_{1}\right), \quad \frac{\partial M}{\partial b}=\frac{\gamma p_{0}(b)}{I_{0}^{2}}\left(b I_{0}-I_{1}\right) .
$$

Из этих формул видно, что функция $M(a, b)$ не имеет критических точек при конечньх $a$ и $b, a<b$. Далее, на всякой прямой $a=\bar{a}$ (включая $\bar{a}=-\infty)$ производная $\partial M / \partial b$ может обратиться в нуль только один раз, так как функция $h(b)=b I_{0}(\bar{a}, b)-I_{1}(\bar{a}, b)$ монотонно возрастает: $h^{\prime}(b)=I_{0}(\bar{a}, b)>0$. Если $\gamma>0$, то минимальное значение $m_{-}$ функции $M(a, b)$ достигается при $a=-\infty, b=b_{-}$. В силу монотонности $h(b)$ значение $b_{-}$определено однозначно. Аналогично, максимальное значение $m_{+}$достигается при $a=a_{+}, b=+\infty$.

ЛЕмма 1. Пусть $\gamma>0 u m_{-}<m<0$. Тогда мнохсество уровня $E_{m}=\{M(a, b)$ $=m\}$ есть гладкая кривая, имеющая две горизонтальные асимптоты $b=b_{1} u$ $b=b_{2}$. При этом $b_{1}<m<b_{2}$.

ДокАЗАТЕЛЬСтво. Уравнение множества $E_{m}$ есть $I_{1}(a, b)=m I_{0}(a, b)$. Вьписав его явно (см. (2.4)), получим при $m<0$

$$
\int_{a}^{b} x p_{0}(x) d x<m \int_{a}^{b} p_{0}(x) d x
$$

Отсюда $a<m$ для всех точек $E_{m}$. В окрестности любой точки $E_{m}$ с конечными $a$ и $b$ это множество есть дуга гладкой кривой (поскольку $d M(a, b) \neq 0$ ). Дифференцируя уравнение $E_{m}$, получим

$$
a^{\prime}(b)=\frac{p_{0}(b)(m-b)}{p_{0}(a)(m-a)} .
$$

Пусть $b<m$ в некоторой точке $\left(a_{0}, b_{0}\right) \in E_{m}$. Тогда в этой точке $a^{\prime}(b)>0$. При движении по кривой $E_{m}$ в сторону увеличения $b$ производная $a^{\prime}(b)$ должна сменить знак (иначе, $a(b)$ будет расти и превзойдет значение $m$ ). Ветвь $E_{m}$, расположенная при $b>m$, уходит неограниченно влево. На ней $b$, продолжая возрастать, стремится к конечному пределу $b_{2}$ (в противном случае $M(a, b)$ при $a \rightarrow-\infty$ и $b \rightarrow+\infty$ на этой ветви стремилось бы к нулю). Аналогично, на нижней ветви, левее точки $\left(a_{0}, b_{0}\right), b$ стремится к $b_{1}<m$. Описанная картина, конечно, не зависит от выбора "начальной" точки $\left(a_{0}, b_{0}\right)$. Поскольку на каждой прямой $a=\bar{a}$ может быть не более двух точек, в которых $M(a, b)=m$, то других связных компонент в множестве $E_{m}$ нет.

ДоБАвлЕНИЕ. Вполне аналогично устроено множество уровня $E_{m}$ при $m>0: E_{m}$ есть гладкая кривая с вертикальньпи асимптотами $a=a_{1}$ и $a=a_{2}$; при этом $a_{1}<$ $m<a_{2}$. На особом положении находится множество $E_{0}$, задаваемое уравнением $I_{1}(a$, $b)=0$ (см. $(2.4 b))$. Оно состоит из прямой $a=b$ и кривой, выходящей из точки $(0,0)$, при движении по которой (влево) $a \rightarrow-\infty$ и $b \rightarrow+\infty$. 
8. Лемма 2. На всем семействе функций (2.2) функиионал $M[w]$ меняется в тех же пределах, что на ступенчатых функииях.

ДокАЗАТЕЛЬСТво. Пусть минимум $M(a, b)$ достигается при $a=-\infty, b=b_{-}$. Приравняв нулю производную $M(-\infty, b)$ (см. начало п. 7 ), получим $m_{-}=b_{-}$. Пусть $w_{-}(x)$ - ступенчатая функция, отвечающая $a=-\infty$ и $b=b_{-}$, а $w(x)$ - произвольная функция, удовлетворяющая неравенствам (2.2) и отличная от $w_{-}(x)$ на множестве положительной меры. При сделанном выборе

$$
\int_{-\infty}^{+\infty}\left(w_{-}(x)-w(x)\right)\left(x-b_{-}\right) p_{0}(x) d x<0,
$$

откуда, обозначив $I_{k-}=I_{k}\left[w_{-}\right]$и $I_{k}=I_{k}[w]$, имеем

$$
I_{1-}-I_{1}-b_{-}\left(I_{0-}-I_{0}\right)<0 \text {. }
$$

Подставив сюда $b_{-}=m_{-}=I_{1-} / I_{0-}$ и поделив на $I_{0}$, получим $M[w]>M\left[w_{-}\right]=m_{-}$.

Аналогично доказьвается, что $M[w] \leqslant m_{+}$для любой $w(x)$. Равенство возможно лишь при совпадении $w(x)$ со ступенчатой функцией $w_{+}(x)$ (почти всюду).

9. Условные экстремумы $D(a, b)$. Пусть снова $\gamma>0$. Фиксируем значение $m<0$ $\left(m>m_{-}\right)$и рассмотрим функцию $I_{2}(a, b) / I_{0}(a, b)$ на линии $E_{m}$. Положим $\psi(b)=$ $I_{2}(a(b), b) / I_{0}(a(b), b)$. Используя лемму 1 , легко проверить (см. формулы $(2.4)$ и $\left.(7.1)\right)$, что при $a \ll-1$ производная $\psi$ отрищательна на нижней ветви кривой $E_{m}$ и положительна на верхней, так что при $a \rightarrow-\infty$ функция $\psi(b)$ возрастает. Поэтому нижняя грань на $E_{m}$ функции $I_{2} / I_{0}$ (тем самым, и функции $D$ ) достигается во внутренней точке-при конечных $a$ и $b$. В точке минимума $\psi^{\prime}\left(b_{*}\right)=0$. Это условие сводится к равенству

$$
I_{2 *}-\left(a_{*}+b_{*}\right) I_{1 *}+a_{*} b_{*} I_{0 *}=0,
$$

здесь $I_{k *}=I_{k}\left(a_{*}, b_{*}\right)$. Случаи $m>0\left(m<m_{+}\right)$и $m=0$ разбираются аналогично. Равенство (9.1) для экстремальных точек $D(a, b)$ на кривой $E_{m}$ остается в силе.

10. Об условном экстремуме функционала $D[w]$. Рассмотрим совокупность функций $w(x)$, удовлетворяющих неравенствам (2.2).

Фиксируем число $m$ из области значений функционала $M[w]$.

ТЕОрема 2. Пусть $p_{0}(x)>0$ при всех $x$. Тогда нижняя грань функиионала $D[w]$ на множестве функиий $w(x)$, для которых $M[w]=m$, достигается на ступенчатой функиии вида (2.3) с $\gamma>0$. Экстремальная функиия определена однозначно.

ДоБАвлЕниЕ. Для экстремальной ступенчатой функции $a=-\infty$ при $m=m_{-}$, $b=+\infty$ при $m=m_{+}$; для остальных допустимых $m$ значения $a$ и $b$ конечны.

ДокАЗАТЕЛЬСТво. При $m=m_{-}$ограничению $M[w]=m$ в силу леммы 2 удовлетворяет только (единственная) ступенчатая функция, и утверждение теоремы становится тривиальньм. То же верно при $m=m_{+}$. Пусть теперь $m_{-}<m<m_{+}$и минимум функции $D(a, b)$ при условии $M(a, b)=m$ достигается в точке $\left(a_{*}, b_{*}\right)$. Пусть $w_{*}(x)-$ соответствующая ступенчатая функция, а $w(x)$ - произвольная функция семейства $(2.2)$, для которой $M[w]=m$. Рассмотрим снова интеграл (5.1); он неположителен. Раскрывая скобки, получим, используя обозначения п. 5 и равенство (9.1),

$$
-I_{2}+\left(a_{*}+b_{*}\right) I_{1}-a_{*} b_{*} I_{0} \leqslant 0 .
$$

Поделим это неравенство на $I_{0}$, а равенство (9.1) на $I_{0 *}$ и сложим. Поскольку $I_{1} / I_{0}=$ $I_{1 *} / I_{0 *}(=m)$, получим $I_{2 *} / I_{0 *}-I_{2} / I_{0} \leqslant 0$ или $D\left[w_{*}\right] \leqslant D[w]$. Знак равенства возможен, только если $w_{*}(x)=w(x)$ почти всюду. 
СЛЕДСТВИЕ. Нижняя грань функционала $D[w]$ на всем семействе функиий (2.2) та же, что на подмножестве ступенчатых функиий, она совпадает с минимумом функиии $D(a, b)$.

(Напомним, что этот минимум достигается при конечных $a$ и $b$; см. п. 4.)

Таким образом, второе утверждение теоремы 1 есть следствие теоремы 2.

ЗАмечание 4. Ступенчатых функций, доставляющих минимум $D[w]$ (на всем семействе функций (2.2)), может быть несколько. Простейший пример строится так. Пусть функция $p_{0}(x)$ четная и ее график состоит из двух узких высоких пиков. Если $\gamma$ достаточно велико, то наименьшая дисперсия распределения $p(x)$ получится при $w(x)$ равной $1+\gamma$ на одном из пиков и 1 в остальных точках. Здесь, стало быть, есть две экстремальные ступенчатые функции.

ЗАмечАниЕ 5 . Задача о максимуме $D[w]$ при заданном значении $M[w]$ приводит к аналогичному ответу: максимум достигается на ступенчатой функции.

11. Произвольное исходное распределение. Если $p_{0}(x)$ где-то обращается в нуль, то основные утверждения теоремы 1 остаются в силе: верхняя и нижняя грани дисперсии модифицированной плотности $p(x)$ (см. п. 1) достигаются и притом на ступенчатых функциях $w(x)$. В этом легче всего убедиться предельньм переходом от положительных $p_{0}(x)$, которьй мы сейчас наметим для максимума $D$. Что касается утверждения о единственности экстремальной $w(x)$ для верхней грани $D$, то оно, конечно, перестает быть верным в буквальном смысле: значения $w(x)$ там, где $p_{0}(x)$ равно нулю, могут быть произвольньпи. Однако легко доказать, используя простые соображения выпуклости (приведенные ниже), что если в рассматриваемом классе распределение $p(x)$ с максимальной дисперсией существует, то оно единственно.

Итак, пусть задана произвольная непрерывная плотность $p_{0}(x) \geqslant 0$. Пусть $q(x)-$ непрерьвная положительная плотность (с конечным средним значением и дисперсией). Положим

$$
p_{\varepsilon}(x)=(1-\varepsilon) p_{0}(x)+\varepsilon q(x)
$$

Обозначим через $I_{k \varepsilon}$ функционалы $I_{k}$, отвечающие распределению $p_{\varepsilon}(x)$ (см. (1.2)), и рассмотрим класс функций $w(x)$, определяемьй неравенствами (2.1). При $\varepsilon \rightarrow 0$ $I_{k \varepsilon}[w] \rightarrow I_{k 0}[w]$ равномерно на всем этом классе. Поскольку (при любой $\left.p(x)\right) I_{0}[w] \geqslant$ $1+\gamma$, то при $\varepsilon \rightarrow 0$ функционалы $D_{\varepsilon}[w]$ равномерно стремятся к $D_{0}[w]$ и $\sup D_{\varepsilon} \rightarrow \sup D_{0}$. При каждом $\varepsilon>0$ справедлива теорема 1: существует ступенчатая функция $w_{* \varepsilon}(x)$, на которой достигается верхняя грань $D_{\varepsilon}$. Выберем последовательность значений $\varepsilon_{j}$, стремящуюся к нулю, пусть $a_{j}$ и $b_{j}$ определяют соответствуюшие ступенчатые функции $w_{* j}(x)$. Очевидно, $D_{0}\left[w_{* j}\right] \rightarrow \sup D_{0}$ при $j \rightarrow \infty$. Выберем подмножество индексов $j$ так, чтобы $a_{j}$ и $b_{j}$ стремились к пределам $a$ и $b$ (допуская, что $a=-\infty$ или $b=+\infty$ ). Для ступенчатой функции $w_{*}(x)$, отвечающей этим $a$ и $b, D_{0}\left[w_{*}\right]=\sup D_{0}$.

12. Единственность распределения $p(x)$ с максимальной дисперсией. Дисперсия $D$, взятая с обратньм знаком, в ее зависимости от плотности вероятностного распределения есть вьпукльй функционал:

$$
D\langle p\rangle \geqslant \frac{1}{2}\left(D\left\langle p_{1}\right\rangle+D\left\langle p_{2}\right\rangle\right) \quad \text { при } p=\frac{1}{2}\left(p_{1}+p_{2}\right) .
$$


Поэтому соображения вьпуклости (не использованные явно до сих пор) полезны в нашей задаче. Рассмотрим совокупность $W$ всех функций $w(x)$, удовлетворяющих неравенствам (2.1); $W$ - вьпуклое множество. Фиксируем функцию $p_{0}(x)$ (не предполагая более, что она везде положительна) и рассмотрим модифицированные распределения $p(x)$ при всех $w(x) \in W$. Совокупность $P$ функций $p(x)$ - тоже вьпуклое множество. Мы изучаем отображение $W$ в $P$, задаваемое формулой $p(x)=w(x) p_{0}(x) / I_{0}[w]$. При этом отображении отрезок, соединяющий два элемента $W$, переходит в отрезок, соединяюший образы. Более точно, если

$$
w=\alpha w_{1}+(1-\alpha) w_{2}, \quad 0<\alpha<1,
$$

то $p=\beta p_{1}+(1-\beta) p_{2}$, где

$$
\beta(\alpha)=\frac{\alpha I_{01}}{\alpha I_{01}+(1-\alpha) I_{02}}, \quad I_{0 k}=I_{0}\left[w_{k}\right] .
$$

Крайние точки множества $W$ (не представимые в виде $(12.2))$ - функции $w(x)$, принимающие (почти всюду) только два значения: 1 и $1+\gamma$. Пусть $S$-множество тех $x$, при которых $p_{0}(x)>0$. Крайние точки в множестве $P$ - функции $p(x)$, для которых $w(x)$ принимает только (те же) два значения на множестве $S$. Для краткости назовем внутренними все элементы $P$, не являющиеся крайними.

ЗАмЕчАниЕ 6 . По заданному $p(x)$ значения $w(x)$ на $S$, вообще говоря, не восстанавливаются однозначно - умножение $w$ на константу не сказывается на $p$. Но если $w(x)$ принимает на $S$ (почти всюду) только значения 1 и $1+\gamma$, то эта неопределенность исчезает.

ЛЕмма 3. Пусть функиия $w(x)$, удовлетворяющая неравенствам (2.1), принимает промежуточные значения (из интервала $1+\gamma<w<1$ ) на подмножестве $S_{1}$ множества $S$, имеющем полохительную меру. Тогда на $w$ не может достигаться верхняя грань функиионала $D[w]$.

ДокАЗАТЕЛЬСтво. Пусть при некотором $c>0$ на множестве $S_{2}$ положительной меры $\left(S_{2} \subset S_{1}\right)$ выполнены неравенства $1+\gamma+c \leqslant w(x) \leqslant 1-c$. Рассмотрим ограниченные функции $\tau(x)$, равные нулю вне $S_{2}$. Положим $w_{\varepsilon}(x)=w(x)+\varepsilon \tau(x)$. Тогда $D\left[w_{\varepsilon}\right]=D[w]+\varepsilon L[\tau]+O\left(\varepsilon^{2}\right)$, где

$$
L[\tau]=\int_{-\infty}^{+\infty} Q(x) \tau(x) p_{0}(x) d x, \quad Q(x)=A x^{2}+B x+C .
$$

Здесь при заданной функции $w(x) A, B$ и $C$ фиксированы $\left(A=1 / I_{0}[w]>0\right)$. Выберем теперь $\tau(x)$ так, чтобы было $L[\tau] \neq 0$. Например, можно положить $\tau(x) \equiv 1$ на той части множества $S_{2}$, где многочлен $Q(x)$ сохраняет знак, и равной нулю в остальных точках. При малых $\varepsilon$ функции $w_{\varepsilon}(x)$ удовлетворяют неравенствам $(2.1)$ и $D\left[w_{\varepsilon}\right]>D[w]$ при $\operatorname{sign}(\varepsilon L[\tau])>0$.

ЗАмЕчАнИЕ 7. На функциях $w$, удовлетворяющих предположениям леммы 3 , не может также достигаться нижняя грань функционала $D$.

Лемма 4. Верхняя грань дисперсии на множестве $P$ не мохет достигаться во внутренней точке $P$. 
ДокАЗАТЕЛЬСтвО. Пусть $p=\beta p_{1}+(1-\beta) p_{2}$ при $0<\beta<1$ и $p_{1} \neq p_{2}$. Пусть $p_{k}(x)=w_{k}(x) p_{0}(x) / I_{0}\left[w_{k}\right]$. Функции $p(x)$ можно сопоставить $w(x)$, где $w$ задается формулой (12.2) (при этом $\alpha$ находится из (12.3)). Если одна из функций $w_{k}$ принимает промежуточные значения на множестве положительной меры, то этим свойством будет обладать и $w$, что противоречит лемме 3 . Если же функции $w_{1}(x)$ и $w_{2}(x)$ принимают лишь крайние значения, то они различаются на множестве $S_{1} \subset S$, имеющем положительную меру; иначе $p_{1}(x)$ и $p_{2}(x)$ совпадали бы почти всюду. На $S_{1}$ функция $w(x)$ принимает промежуточные значения. По лемме 3 на $w$ не может достигаться верхняя грань $D[w]$, т.е. дисперсия $D\langle p\rangle$ не максимальна.

ПРЕДЛОЖЕНИЕ. В классе $P$ может существовать не более одной функиии, на которой достигается верхняя грань функиионала $D\langle p\rangle$.

ДокАЗАтЕльство. Предположим, что значение $\bar{D}=\sup D\langle p\rangle$ достигается на функциях $p_{1}(x)$ и $p_{2}(x)$, различающихся на множестве положительной меры. Пусть им соответствуют $w_{1}(x)$ и $w_{2}(x)$ (принимаюшие лиш два значения в силу леммы 3 ). Положим $2 p(x)=p_{1}(x)+p_{2}(x)$. Для $p$ можно выбрать $w$ по формуле (12.2). Следовательно, $w(x)$ принимает промежуточные значения на множестве положительной меры (где не совпадают $w_{1}$ и $\left.w_{2}\right)$. По лемме $4 D[w]=D\langle p\rangle$ не может быть максимальньп, но в силу формулы (12.1) $D\langle p\rangle=\bar{D}$. Полученное противоречие доказьвает утверждение.

13. Приложение. Следующий комментарий сообщен автору А.С. Кондрашовьм (упомянутым в начале статьи):

ПроБЛЕмА. Со времен Дарвина видообразование, т.е. процесс распада одной популячии на две, является иентральным оббектом рассмотрения в әволючионной биологии. Для изучения видообразования полезно знать, какая из возмохсных функиий отбора данной интенсивности оказывает наиболее сильное разрывающее воздействие на популячию.

Этот вопрос приводит к математической задаче, сформулированной в п. 1 (точнее к ее первой части - вопросу о максимуме дисперсии).

Смысл всех величин в исходной, содержательной задаче таков:

$x$ - значение некоторого количественного признака;

$p_{0}(x)$ - распределение этого признака среди особей выбранной популящии (численность ее предполагается достаточно большой, чтобы имело смысл рассматривать непрерывные распределения);

$w(x)$ - “функция отбора", характеризующая выживаемость особей с данным значением выбранного признака (предполагается, что только от него выживаемость и зависит!);

$p(x)$ - распределение того же признака после действия отбора.

Постоянная $K$ характеризует интенсивность отбора.

Институт математических проблем биологии $\mathrm{PAH}$,

Поступило

г. Пущино Московской обл.

08.10.1999

shnol@impb.psn.ru

Исправленный вариант

29.05.2001 